\title{
Electron beam - plasma interaction in a dusty plasma with excess suprathermal electrons
}

\author{
A. Danehkar, ${ }^{1}$ N. S. Saini, ${ }^{2}$ M. A. Hellberg, ${ }^{3}$ and I. Kourakis ${ }^{4}$ \\ ${ }^{1}$ Department of Physics and Astronomy, \\ Macquarie University, Sydney, NSW 2109, Australia \\ ${ }^{2}$ Department of Physics, Guru Nanak Dev University, Amritsar-143005, India \\ ${ }^{3}$ School of Physics, University of KwaZulu-Natal, Durban 4000, South Africa \\ ${ }^{4}$ Department of Physics and Astronomy, \\ Queen's University Belfast, BT7 1NN, UK
}

\begin{abstract}
The existence of large-amplitude electron-acoustic solitary structures is investigated in an unmagnetized and collisionless two-temperature dusty plasma penetrated by an electron beam. A nonlinear pseudopotential technique is used to investigate the occurrence of stationary-profile solitary waves, and their parametric dependence on the electron beam and dust perturbation is discussed.

PACS numbers: 52.27.Lw, 52.35.Sb, 52.35.Mw, 52.40.-w
\end{abstract}

Keywords: Dusty (complex) plasmas, solitons, nonlinear phenomena, plasma interactions

We have previously studied electron-acoustic solitary waves in the presence of a suprathermal electron component. [1] Our aim here is to investigate the effect of beam electrons and dust on the electrostatic solitary structures.

We consider a plasma consisting of cold inertial drifting electrons (the beam), cold inertial background electrons, hot suprathermal electrons modeled by a kappa-distribution, stationary ions, and stationary dust (of either positive or negative charge). The dynamics of the cold inertial background electrons and the beam electrons are governed by the following normalized one-dimensional equations:

$$
\begin{gathered}
\frac{\partial n}{\partial t}+\frac{\partial(n u)}{\partial x}=0, \frac{\partial u}{\partial t}+u \frac{\partial u}{\partial x}=\frac{\partial \phi}{\partial x} \\
\frac{\partial n_{b}}{\partial t}+\frac{\partial\left(n_{b} u_{b}\right)}{\partial x}=0, \frac{\partial u_{b}}{\partial t}+u_{b} \frac{\partial u_{b}}{\partial x}=\frac{\partial \phi}{\partial x} \\
\frac{\partial^{2} \phi}{\partial x^{2}}=-(\eta+s \delta)+n+\beta n_{b}+(\eta+s \delta-1-\beta)\left(1-\frac{\phi}{\left[\kappa-\frac{3}{2}\right]}\right)^{-\kappa+1 / 2} .
\end{gathered}
$$



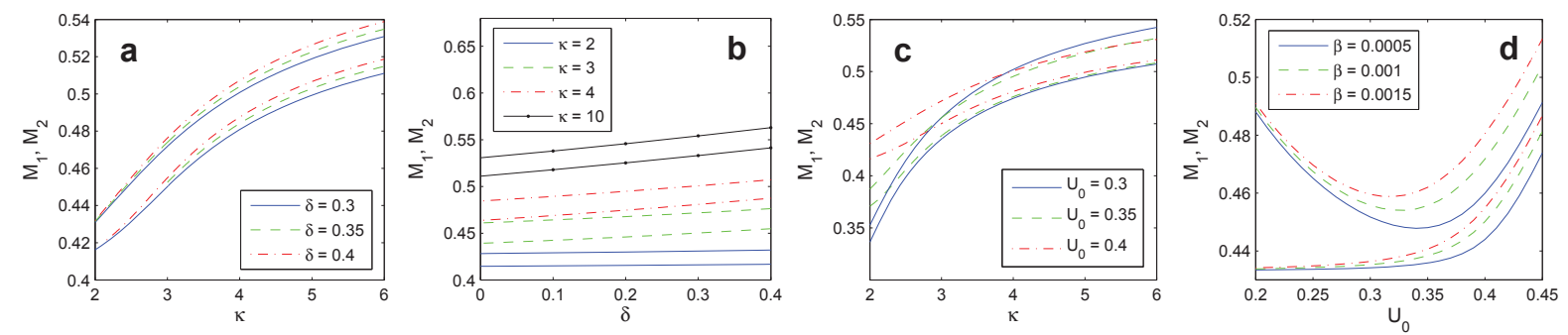

FIG. 1: Soliton existence region $\left(M_{1}<M<M_{2}\right)$ : (a) versus $\kappa$ for different $\delta$ values; (b) versus $\delta$ for different $\kappa$ values; (c) versus $\kappa$ for different $U_{0}$ values; (d) versus $U_{0}$ for different $\beta$ values. The remaining values are $\kappa=4.5, \delta=0.3, s=-1, \beta=0.001, U_{0}=0.4$ and $\eta=4.5$, unless values are given.

Here, $n$ and $n_{b}$ denote the fluid density variables of the cool electrons and the beam electrons normalized with respect to $n_{c, 0}$ and $n_{b, 0}$. The velocities $u$ and $u_{b}$, and the equilibrium beam speed $U_{0}=u_{b, 0} / c_{t h}$ are scaled by the hot electron thermal speed $c_{t h}=\left(k_{B} T_{h} / m_{e}\right)^{1 / 2}$, and the wave potential $\phi$ by $k_{B} T_{h} / e$. Time and space are scaled by the plasma period $\omega_{p c}^{-1}=\left(n_{c, 0} e^{2} / \varepsilon_{0} m_{e}\right)^{-1 / 2}$ and the characteristic length $\lambda_{0}=\left(\varepsilon_{0} k_{B} T_{h} / n_{c, 0} e^{2}\right)^{1 / 2}$, respectively. We define the hot-to-cold electron charge density ratio $\alpha=n_{h, 0} / n_{c, 0}$, the beam-to-cold electron charge density ratio $\beta=n_{b, 0} / n_{c, 0}$, the ion-to-cold electron charge density ratio $\eta=Z_{i} n_{i, 0} / n_{c, 0}$, and the dust-to-cold electron charge density ratio $\delta=Z_{d} n_{d, 0} / n_{c, 0}$. Here, suprathermality is denoted by the spectral index $\kappa$, and $s= \pm 1$ is the sign of the dust charge for positive or negative dust grains. At equilibrium, the plasma is quasi-neutral, so $\eta+s \delta=1+\alpha+\beta$.

Anticipating constant profile solutions of Eqs. (11)-(3) in a stationary frame traveling at a constant normalized velocity $M$, implying the transformation $\xi=x-M t$, we obtain $n=\left(1+2 \phi / M^{2}\right)^{-1 / 2}$ and $n_{b}=\left[1+2 \phi /\left(M-U_{0}\right)^{2}\right]^{-1 / 2}$. Substituting in Poisson's equation and integrating yields a pseudo-energy balance equation $\frac{1}{2}(d \phi / d \xi)^{2}+\Psi(\phi)=0$, where the Sagdeev pseudopotential $\Psi(\phi)$ reads

$$
\begin{aligned}
& \Psi(\phi)=(\eta+s \delta) \phi+M^{2}\left(1-\left[1+2 \phi / M^{2}\right]^{\frac{1}{2}}\right)+\beta\left(M-U_{0}\right)^{2} \times \\
& \left(1-\left[1+2 \phi /\left(M-U_{0}\right)^{2}\right]^{\frac{1}{2}}\right)+(\eta+s \delta-1-\beta)\left(1-\left[1-\phi /\left(\kappa-\frac{3}{2}\right)\right]^{-\kappa+\frac{3}{2}}\right) .
\end{aligned}
$$

Reality of the density variable implies two limits on the electrostatic potential $\phi_{\max }=-M^{2} / 2$ and $-\left(M-U_{0}\right)^{2} / 2$ for $U_{0}<0$ and $U_{0}>0$, respectively. In order for solitary waves to exist, 
two constraints must be satisfied, i.e., $F_{1}(M)=-\left.\Psi^{\prime \prime}(\phi)\right|_{\phi=0}>0$ and $F_{2}(M)=\left.\Psi(\phi)\right|_{\phi=\phi_{\max }}>$ 0 , which yield the solutions for the lower and upper limit in $M$.

As shown in Figure 1, the existence domain for solitons becomes narrower with increasing suprathermal excess (decreasing $\kappa$ ), increasing equilibrium beam speed, and decreasing beam density. Dust charge density shows little effect on the width of the existence domain, but for quasi-Maxwellian electrons, it weakly increases the typical values of $M$. It was found that both increasing $\kappa$ and increasing negative dust charge density significantly reduce soliton amplitude at fixed $M$ (not shown here).

\section{ACKNOWLEDGMENTS}

AD, NSS and IK thank the Max-Planck Institute for Extraterrestrial Physics for their support. IK acknowledges support from UK EPSRC via S\&I grant EP/D06337X/1.

[1] A. Danehkar, N. S. Saini, M.A. Hellberg and I. Kourakis, Physics of Plasmas 18, 072902 (2011). 